\title{
Phase-matched nondegenerate four-wave mixing in one-dimensional photonic crystals
}

\author{
C. Becker, ${ }^{\text {a) }}$ M. Wegener, S. Wong, and G. von Freymann \\ Institut für Nanotechnologie, Forschungszentrum Karlsruhe in der Helmholtz-Gemeinschaft, Postfach 3640, \\ D-76021 Karlsruhe, Germany and DFG-Center for Functional Nanostructures, Universität Karlsruhe \\ (TH), D-76128 Karlsruhe, Germany and Institut für Angewandte Physik, Wolfgang-Gaede-Straße 1, \\ Universität Karlsruhe (TH), D-76131 Karlsruhe, Germany
}

(Received 27 July 2006; accepted 10 August 2006; published online 29 September 2006)

\begin{abstract}
The authors report on nondegenerate four-wave mixing in the near infrared using a one-dimensional chalcogenide-glass based photonic crystal. For 76 lattice constants, they find a 3.5-fold enhancement of the mixing signal with respect to the optimum-thickness bulk chalcogenide film. The key is the ability to tailor the dispersion relation of light in the photonic crystal, allowing for phase matching. Numerical calculations agree well with the experiments. (c) 2006 American Institute of Physics. [DOI: $10.1063 / 1.2358295]$
\end{abstract}

Photonic crystals are a class of artificial tailored materials for optics and photonics. They are usually composed of two different dielectrics, which are periodically arranged along $d=1,2$ or 3 dimensions. The periodicity leads to Bloch waves and a photonic band structure, which allows for tailoring the effective material dispersion relation of light. This aspect immediately suggests applications in nonlinear optics, where phase matching is known to be of crucial importance for efficient frequency conversion. Indeed, photonic crystals enable larger conversion efficiencies compared to the constituent bulk materials - as already pointed out theoretically by Bloembergen and Sievers in 1970 (Ref. 1) and demonstrated experimentally by van der Ziel and Ilegem in 1976 (Ref. 2) using a one-dimensional (1D) photonic crystal. More recent experimental examples are phase-matched direct third-harmonic generation (THG) in three-dimensional polymer-based opal structures, ${ }^{3}$ THG in 1D liquid-crystal structures, ${ }^{4}$ and phase-matched second-harmonic generation (SHG) in 1D II-VI semiconductor-based photonic crystals., Surface SHG has been accomplished from two-dimensional III-V semiconductor-based photonic crystals. ${ }^{7}$ In all these degenerate experiments, only one wave with a single color impinges onto the sample. In this case, phase matching requires simply matching the effective index at the fundamental frequency to that of the harmonic.

Tailoring the dispersion relation of light in 1D photonic crystals also allows for phase matching in nondegenerate processes, i.e., nonlinear mixing with different incident colors. Here, we realize phase matching for a third-order process of the type $\omega_{3}=2 \omega_{1}-\omega_{2}$ that has not been published so far, to the best of our knowledge.

We employ one-dimensional quarter-wave stacks (1D photonic crystals) composed of a large number of chalcogenide-glass ${ }^{8}$ layer pairs. Chalcogenides are chosen because of their known large nonlinear-optical coefficients $^{9,10}$ and the ease of fabrication. The $1 \mathrm{D}$ configuration is employed because it conveniently allows for many tens of periods, corresponding to sample thicknesses exceeding the bulk phase-matching coherence length. We consider two different samples, one with 52 periods (104 individual

\footnotetext{
${ }^{a)}$ Electronic mail: christiane.becker@physik.uni-karlsruhe.de EVA-STAR (Elektronisches Volltextarchiv - Scientific Articles Repository) 0003-6951/2006/89(13)/131122/3/\$23.00://digbib.ubka.uni-karlsruhe de/volltexte/1000010963 ter wavelength is accompanied by changes in the pulse du-
} Downloaded 18 Mar 2009 to 129.13.72.198. Redistribution subject to AlP license or copyright; see http://apl.aip.org/apl/copyright.jsp layers), the other with 76 periods (152 individual layers). Both samples have been fabricated by thermal evaporation of granular $\mathrm{As}_{2} \mathrm{Se}_{3}$ and $\mathrm{As}_{2} \mathrm{~S}_{3}$ in a high-vacuum chamber at pressures below $10^{-5}$ mbar and temperatures around 470 and $365^{\circ} \mathrm{C}$, respectively. The nominal $\mathrm{As}_{2} \mathrm{Se}_{3}$ and $\mathrm{As}_{2} \mathrm{~S}_{3}$ layer thicknesses are 106 and $125 \mathrm{~nm}$, respectively. The lattice constant is the sum, i.e., $a=231 \mathrm{~nm}$ [see inset in Fig. 1(a)]. Well below their absorption edge, the dispersion of the chalcogenides can be described by a dielectric function corresponding to a single undamped oscillator (without employing the rotating-wave approximation), i.e., by

$$
\epsilon(\omega)=\epsilon_{b}-b\left(\frac{1}{\omega-\omega_{0}}+\frac{1}{\omega+\omega_{0}}\right) .
$$

The resulting refractive index is $n(\omega)=\sqrt{\epsilon(\omega)}$. For the $\mathrm{As}_{2} \mathrm{Se}_{3}$, we use $\epsilon_{b}=4.5, \quad b=4.441 \mathrm{eV} / \hbar, \quad$ and $\omega_{0}$ $=2.812 \mathrm{eV} / \hbar$; for the $\mathrm{As}_{2} \mathrm{~S}_{3}, \epsilon_{b}=3.55, b=3.873 \mathrm{eV} / \hbar$, and $\omega_{0}=3.622 \mathrm{eV} / \hbar$. The refractive index of the thick glass substrate is taken as $n_{\mathrm{SiO}_{2}}=1.5$.

Using this material dispersion and employing the "cutting surface method," ${ }^{11}$ we calculate the band structure $k(\omega)$ of the 1D photonic crystal and depict it in Fig. 1(a). The gray area highlights the 1D photonic stop band. The retrieved effective refractive index of the structure, defined by $n_{\text {eff }}(\omega)$ $=k(\omega) c_{0} / \omega$, is shown in (b). $c_{0}$ is the vacuum speed of light. The calculated and the measured normal-incidence transmittance of a 52-period stack are shown in (c) and (d), respectively.

Next, we compute the wave vector mismatch $\Delta k=2 k_{1}$ $-k_{2}-k_{3}=2 k\left(\omega_{1}\right)-k\left(\omega_{2}\right)-k\left(\omega_{3}\right)$ for the generated frequency component $\omega_{3}=2 \omega_{1}-\omega_{2}$ on the basis of the retrieved index and plot it on a gray scale versus the two input frequencies $\omega_{1}$ and $\omega_{2}$ in Fig. 2. This plot gives an overview about the parameter space to be explored below. The white regions correspond to $\Delta k=0$ hence perfect phase matching, i.e., to large nonlinear signals.

In our nonlinear-optical experiments, we employ two optical parametric amplifiers (OPAs), both pumped by a regeneratively amplified Ti:sapphire femtosecond laser system (Spectra Physics Hurricane) and independently tunable in the spectral range of interest. Unfortunately, tuning the OPA cen- 


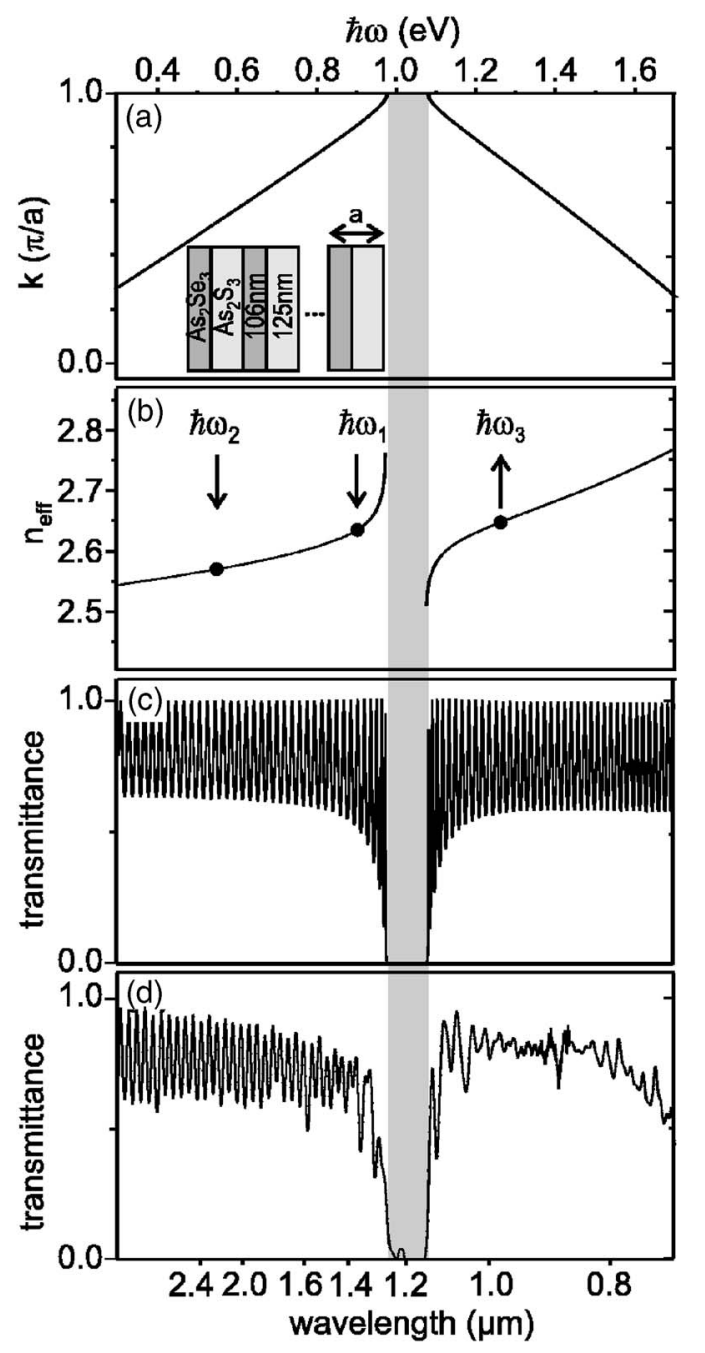

FIG. 1. (a) Calculated band structure of the 1D photonic crystal. (b) Corresponding effective refractive index, (c) calculated, and (d) measured normalincidence transmittances of a 52 period sample. The combination of the three frequencies $\omega_{1}, \omega_{2}$, and $\omega_{3}$ used in Fig. 3 is indicated.

ration $(80-130 \mathrm{fs})$ and in the beam divergence, hence in the spot size on the sample, leading to strong intensity variations. Thus, unambiguous and reproducible experiments require relating the nonlinear signals from the $1 \mathrm{D}$ photonic crystals to some reference. Here, we chose bulk $\mathrm{As}_{2} \mathrm{Se}_{3}$ chalcogenide as this reference. It is known that $\mathrm{As}_{2} \mathrm{Se}_{3}$ chalcogenide glasses have an about sevenfold larger third-order nonlinear susceptibility, $\chi^{(3)}$, than $\mathrm{As}_{2} \mathrm{~S}_{3}{ }^{8-10}$ The two OPA beams at frequencies $\omega_{1}$ and $\omega_{2}$, respectively, are focused collinearly and under normal incidence onto the sample with a $5 \mathrm{~cm}$ focal length lens. By varying the intensity of both incident beams, we have carefully checked that our experiments are performed in the third-order perturbational regime (not shown), i.e., the signal strength is proportional to the intensity of beam No. 2 and proportional to the square of the intensity of beam No. 1. The generated signal at frequency $2 \omega_{1}-\omega_{2}$ is separated from the transmitted fundamentals by a prism and a diaphragm, serving as a spectral filter, and is detected by an InGaAs photodiode.

Figure 3 shows the measured mixing signal $\omega_{3}=2 \omega_{1}$ $-\omega_{2}$ for the bulk $\mathrm{As}_{2} \mathrm{Se}_{3}$ glass film versus its film thickness $L$ (open squares). As expected, one gets phase-matching oscillations. For the particular choice of $\omega_{1}=0.894 \mathrm{eV} / \hbar$ and $\omega_{2}=0.536 \mathrm{eV} / \hbar$, the coherence length obtained from a leastDownloaded 18 Mar 2009 to 129.13.72.198. Redistribution subject

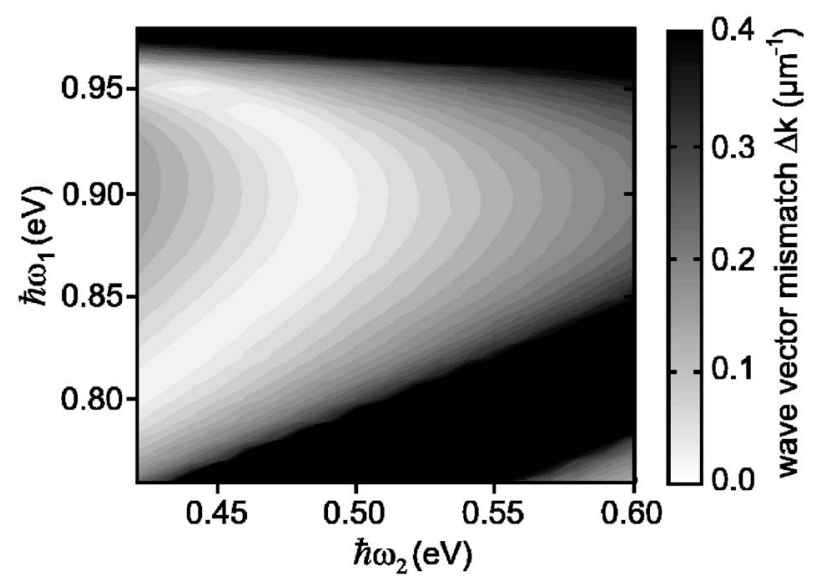

FIG. 2. Wave vector mismatch $\Delta k=2 k_{1}-k_{2}-k_{3}$ for the generated frequency component $\omega_{3}=2 \omega_{1}-\omega_{2}$ as derived from the effective index $n_{\mathrm{eff}}(\omega)$ for the $1 \mathrm{D}$ photonic crystal shown in Fig. 1 on a gray scale as a function of the two incident frequencies $\omega_{1}$ and $\omega_{2}$. The plot for $\Delta k$ for bulk $\mathrm{As}_{2} \mathrm{Se}_{3}$ is essentially black at the frequencies of interest (not shown).

squares fit is $L_{c}=2 / \Delta k=5.04 \mu \mathrm{m}$. From the comparison of this coherence length and the much smaller lattice constants quoted above, it becomes clear that the effects discussed here are not in the regime of quasi-phase-matching but are rather due to phase matching. Also shown in Fig. 3 are the results of the 1D photonic crystals for the same frequency combination (filled squares). Notably, we obtain larger signals from the 1D photonic crystals than the maximum of bulk chalcogenide $\mathrm{As}_{2} \mathrm{Se}_{3}$. The enhancement is 2.4 -fold for 52 periods (total thickness $L=12.0 \mu \mathrm{m}$ ) and is 3.5 -fold for 76 periods (total thickness $L=17.5 \mu \mathrm{m}$ ) of the photonic crystal. The inset reveals a typical spectrum of the enhancement factor $(\mathrm{EF})$ for the 52-period stack with respect to $L=10.2 \mu \mathrm{m} \mathrm{As} \mathrm{Se}_{3}$ (enlarged open square) for fixed $\omega_{2}=0.536 \mathrm{eV} / \hbar$. The pronounced maximum corresponds to $L / L_{c}=\pi$ for the bulk film, hence very small nonlinear signal for the bulk case. The sharp drop on the high-energy side results from the fact that the frequency $\omega_{1}$ is moving into the stop band. Note that the enhancement factor exceeds unity for a broad range of frequencies, showing the robustness of the effect.

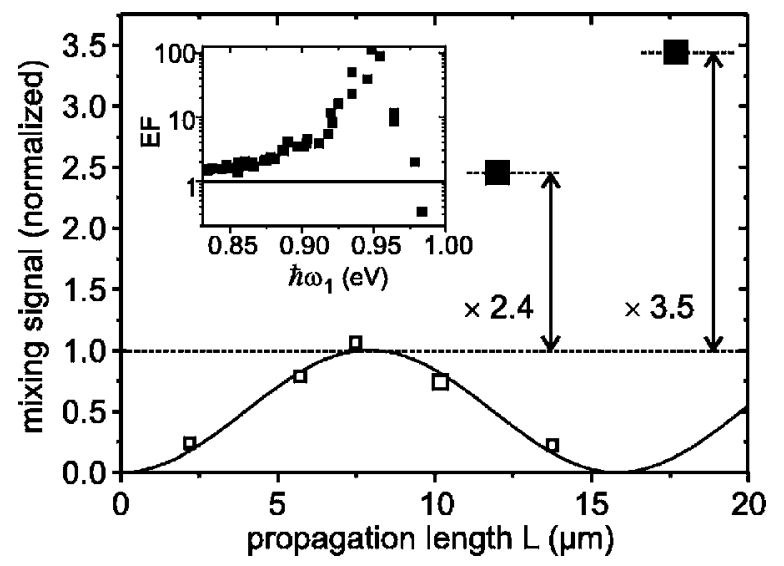

FIG. 3. Measured nondegenerate four-wave mixing signal at frequency $2 \omega_{1}-\omega_{2}$ from bulk $\mathrm{As}_{2} \mathrm{Se}_{3}$ (open squares) and the $1 \mathrm{D}$ photonic crystals with 52 periods and 76 periods (filled squares) for the frequencies $\omega_{1}$ $=0.894 \mathrm{eV} / \hbar$ and $\omega_{2}=0.536 \mathrm{eV} / \hbar$. The signals are normalized to the optimum signal of the bulk film. The inset exhibits a typical spectrum of the enhancement factor (EF) for fixed $\omega_{2}=0.536 \mathrm{eV} / \hbar$ vs $\omega_{1}$ of the 52 period stack compared to a bulk $L=10.2 \mu \mathrm{m}$ thick $\mathrm{As}_{2} \mathrm{Se}_{3}$ film. 


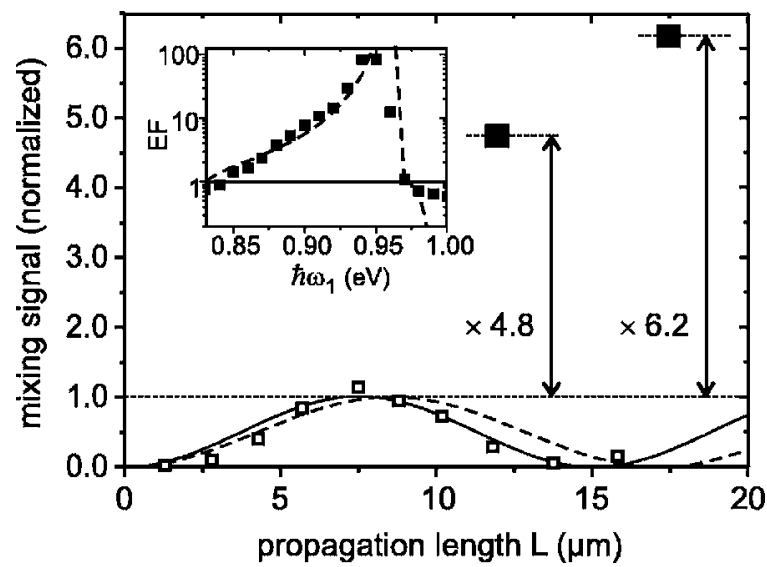

FIG. 4. Theory corresponding to the experiment shown in Fig. 3. The two curves at the bottom compare the results from 1D finite-difference timedomain calculations (squares) and those based on the effective index (dashed curves).

To further strengthen our interpretation, we have also performed numerical one-dimensional finite-difference timedomain calculations $^{12}$ (without employing the rotating-wave approximation) with the parameters quoted above. We neglect the small frequency dependence of the third-order nonlinear susceptibility of the chalcogenide glasses in the spectral range of interest and take the values $\chi^{(3)}=2.9$ $\times 10^{-19} \mathrm{~m}^{2} / \mathrm{V}^{2}$ for $\mathrm{As}_{2} \mathrm{Se}_{3}$ and $\chi^{(3)}=4.2 \times 10^{-20} \mathrm{~m}^{2} / \mathrm{V}^{2}$ for the $\mathrm{As}_{2} \mathrm{~S}_{3}$ glass that we have obtained from $z$-scan measurements and that are in the range of the values published elsewhere (e.g., references in Ref. 8). In our calculations, only the ratio between these values actually enters. Theoretical results arranged as the experiment (Fig. 3) are shown in Fig. 4. Obviously, the overall qualitative agreement is good. Note that theory-in agreement with experiment-shows deviations from the naively expected scaling of the mixing signal proportional to the square of the stack thickness $L$ for perfect phase matching. Intuitively, not all frequency components in the finite-width incident OPA spectra (arising from the about 100 fs pulse duration) fulfill ideal phase matching. Furthermore, note that theory predicts quantitatively larger normalized signals than observed experimentally by about a factor of 2 (compare Figs. 4 and 3). This aspect is likely due to sample imperfections (also see transmittance spectra in Fig. 1).

In conclusion, we have demonstrated an enhancement of the efficiency of nondegenerate four-wave mixing in the near infrared. We obtain an enhancement factor of 3.5 compared to optimum bulk by exploiting the particular dispersion relation of light in a 1D photonic crystal composed of chalcogenide-glass films with 152 layers (76 periods) total. Theory shows that further enhancement of the conversion efficiency could be achieved by increasing the number of layers. Broadly speaking, our results are a new example that tailoring the dispersion relation of light in photonic crystals offers interesting opportunities for phase matching in nonlinear optics.

The authors acknowledge support by the Deutsche Forschungsgemeinschaft (DFG) and the State of BadenWürttemberg through subproject A 1.4 of the Center for Functional Nanostructures (CFN). The research of one of the authors (G.v.F.) is further supported by DFG-project FR 1671/4-3 (Emmy-Noether program).

${ }^{1}$ N. Bloembergen and A. J. Sievers, Appl. Phys. Lett. 17, 483 (1970).

${ }^{2}$ J. P. Van der Ziel and M. Ilegem, Appl. Phys. Lett. 28, 437 (1976).

${ }^{3}$ P. P. Markowicz, H. Tiryaki, H. Pudavar, P. N. Prasad, N. N. Lepeshkin, and R. W. Boyd, Phys. Rev. Lett. 92, 083903 (2004).

${ }^{4}$ P. P. Markowicz, V. K. S. Hsiao, H. Tiryaki, A. N. Cartwrite, P. N. Prasad, K. Dolgaleva, N. N. Lepeshkin, and R. W. Boyd, Appl. Phys. Lett. 87, 05110 (2005).

${ }^{5}$ H. Yang, P. Xie, S. K. Chan, Z. Q. Zhang, I. K. Sou, G. K. L. Wong, and K. S. Wong, Appl. Phys. Lett. 87, 131106 (2005).

${ }^{6}$ H. Yang, P. Xie, S. K. Chan, W. Lu, Z. Q. Zhang, I. K. Sou, G. K. L. Wong, and K. S. Wong, IEEE J. Quantum Electron. 42, 447 (2006).

${ }^{7}$ J. P. Mondia, H. M. van Driel, W. Jiang, A. R. Cowan, and J. F. Young, Opt. Lett. 28, 2500 (2003).

${ }^{8}$ A. Zakery and S. R. Elliott, J. Non-Cryst. Solids 330, 1 (2003).

${ }^{9}$ G. Lenz, J. Zimmermann, T. Katsufuji, M. E. Lines, H. Y. Hwang, S. Splter, R. E. Slusher, S.-W. Cheong, J. S. Sanghera, and I. D. Aggarwal, Opt. Lett. 25, 254 (2000).

${ }^{10}$ J. M. Laniel, N. Hô, R. Vallée, and A. Villeneuve, J. Opt. Soc. Am. B 22 , 437 (2005).

${ }^{11}$ O. Toader and S. John, Phys. Rev. E 70, 046605 (2004).

${ }^{12}$ A. Taflove and S. C. Hagness, Computational Electrodynamics: The Finite-Difference Time-Domain Method, 2nd ed. (Artech House, Boston, 2000), pp. 67-84. 